\title{
VISUAL ATTENTION IN STROKE PATIENTS RETURNING TO DRIVING
}

\author{
Matthew I. Tofield, John P. Wann \\ Action Research Laboratories \\ School of Psychology \\ University of Reading \\ Earley Gate \\ Whiteknights \\ Reading, Berkshire RG6 6AL \\ United Kingdom \\ E-Mail: m.i.tofield@reading.ac.uk
}

\begin{abstract}
The UK along with many European countries lacks a standardised test to objectively test visual attentional deficits in drivers of vehicles, particularly following stroke injury. Detecting motion components in the visual scene may be the first indication of an impending collision, however, this is often overlooked in current assessment procedures. The series of experiments presented here used a semi-immersive virtual reality paradigm and eye gaze monitoring technology, to identify deficits in peripheral processing and extend the concept of useful field of view (UFOV) (Ball et al. 1988; 1993). We used displays that tested peripheral attention with brief (90ms) episodes of relative motion, changing size, luminosity and colour. Participants were presented with different sets of stimuli on a large video screen at $10^{\circ}, 20^{\circ}$, and $30^{\circ}$ eccentricities and used a natural gaze response to look to the cued location. Gaze response was monitored using an ASL 504 eye movement tracking system, and the dependant measure was accuracy of saccade to the eccentric target. Six stroke participants (mean age $=61.16)$ were tested, and their results compared against previously gathered data for the same experiments from three healthy aging groups of drivers (Young: $\mathrm{N}=21$, mean age $=25.29$, Middle aged: $\mathrm{N}=20$, mean age $=50.25$, and Elderly: $\mathrm{N}=20$, mean age $=70.50)$. The systematic decrease in performance with increasing age that was previously found for the healthy aging participants $(\mathrm{p}<0.001)$ was also found in the stroke group, who performed worse than the healthy aging groups in the same tasks $(p<0.001)$. The results underpin our previous finding of deficits in processing basic components of visual control that go beyond current UFOV tests. These current results highlight the compounding effects of stroke injury on the ability of attention to be captured by motion components that may be the first and only indication of an impending collision event. We discuss the implications of treating stroke patients as though they belong to a homogenous group when assessing suitability to return to driving; our results indicate that they clearly do not. Younger stroke drivers made recoveries that placed their performance alongside that of their age-matched healthy controls, unlike older stroke patients who remained more impaired than their age-matched healthy controls. We conclude that assessment of visual attention as it relates to moving objects in a visually cluttered environment should be part of any assessment criteria for measuring fitness to drive in stroke patients making a return to driving.
\end{abstract}

